Ahmed : Effect Of Wet And Dry Cutting Conditions On Dimensional Accuracy In Turning

\title{
Effect Of Wet And Dry Cutting Conditions On Dimensional Accuracy In Turning Operation
}

\author{
Dler Abdullah Ahmed, Assist Lecturer, \\ Technical Institute in Erbil, Mechanical Dept., Erbil-Iraq
}

\begin{abstract}
This work is done by turning a medium carbon steel bare which contains $0.35 \% \mathrm{C}$ using high speed steel cutting tool. Dimensional deviation is taken as a criterion to compare between parts produced by dry and wet cutting condition. It is observed that, in traditional wet cutting condition by water-oil solution, an irregular conical shape is produced and the diameter of the work piece is increased from initial to the end of the work piece. But in the dry cutting condition the diameter is near to the desired diameter because the decrease in the tool length due to a flank wear is substituted due to the thermal expansion of the cutting tool. Fortunately, it is observed that the dry cutting condition is more accurate in diameter than the wet cutting condition.
\end{abstract}

Keywords: Dimensional deviation, Flank wear, Dry and Wet cutting condition.

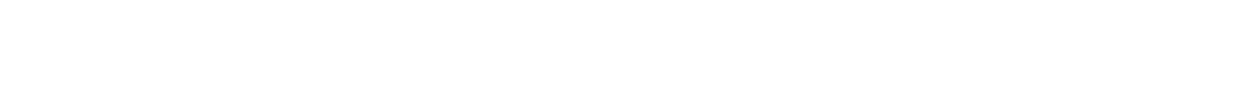

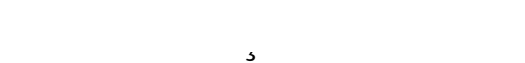

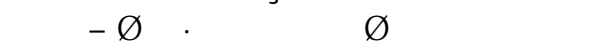

\begin{abstract}
rUீ+ ŤKE

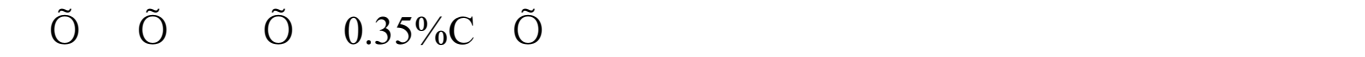

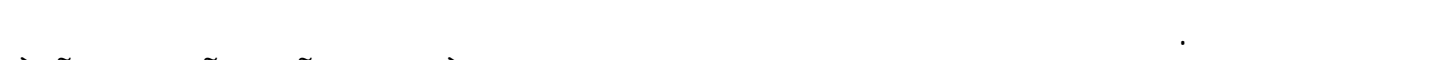

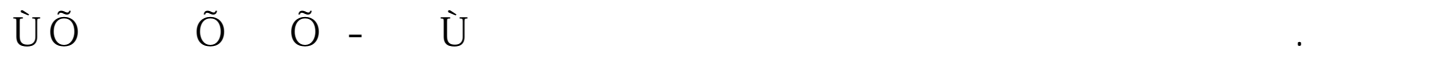

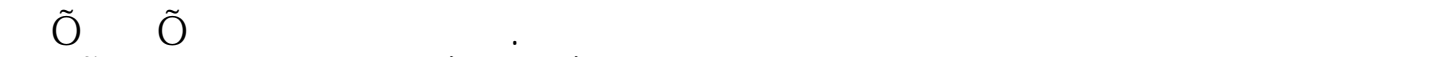

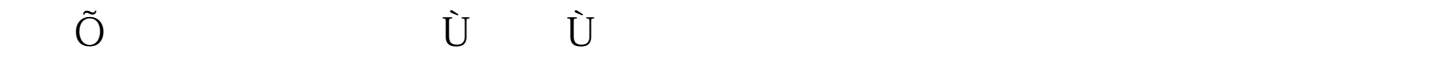

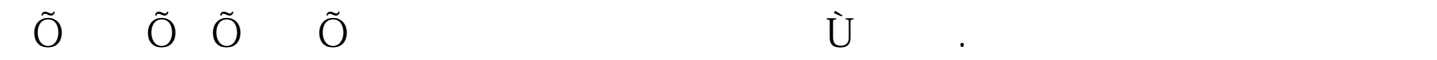
.Rุųũ̂́ 


$\begin{array}{llll}\text { Al-Rafidain Engineering } & \text { Vol.15 } & \text { No.4 } & 2007\end{array}$

\section{Introduction}

Dimensional deviation is significant in turning slender parts. Recently the ultra precision machining will take an even more significant role among other manufactory techniques. Therefore the dimensional accuracy of the machined parts becomes an important criterion of the production [1]. In the other hand, metal machining is usually done using large amounts of cutting fluids. Its total cost has been estimated to be as high as $16 \%$ of total manufacturing costs [2]. Machining is usually characterized by generation of heat and high cutting temperature. At such elevated temperature the cutting tool if not enough hot hard may lose their form stability quickly or wear out rapidly resulting in increased cutting force, dimensional inaccuracy of the product and shorter tool life. The magnitude of this cutting temperature varies with the increase of cutting velocity, feed rate and depth of cut. High cutting zone temperature is conventionally tried to be controlled by employing flood cooling, but it failed to penetrate the chip - tool interface and thus cannot remove heat effectively. Cutting fluids failed to reduce cutting temperature and improve tool life effectively. However, high pressure jet of soluble oil if applied at the chip - tool interface could reduce cutting temperature to some extent [3].

R.Azouzi [4] used cutting fluid and observed that the diameter of the work piece increases gradually from beginning to the end of the work piece. Also, in S.K. Choud [5] the same result is observed, both of them used a cutting fluid and studied the effect of cutting parameters on the dimensional deviation. But, Powel [6] observed that the diameter of the work piece is less than the desired diameter due to the thermal expansion of the cutting tool and work piece and more metal was removed because he worked in dry cutting condition. During machining a slender work piece the nose of the cutting tool is worn due to the friction between the work piece and the tool. This type of wear is called flank wear and a length of the tool is reduced. This decrease of the length of the cutting tool is translated as a positive dimensional deviation on the work piece and the diameter of the work piece is increased. This type of dimensional deviation is called dimensional deviation due to the flank wear. In the other hand, heat and high temperature is generated due to the friction which causes thermal expansion in the tool. This thermal expansion of the cutting tool is translated as a negative dimensional deviation on the work piece and the diameter of the work piece is decreased. Also, some expansion is taken place on the work piece. It is causes more metal removal and smaller diameter of the work piece. The deviation due to the thermal expansion is called thermal error. Xiaoli [7] observed that the thermal error is always in negative and it is presents in dry cutting condition.

\section{Experimental Procedure}

In this work, specimens with $40 \mathrm{~mm}$ in diameter and $300 \mathrm{~mm}$ in length from medium carbon steel, its chemical composition is explained in the Table (1), hardness (168HB30) and Ultimate strength $(618 \mathrm{Mpa})$ was used. It was turned by high speed steel (HSS) cutting tool. Four velocities, four feed rate and one depth of cut were selected as a cutting parameter in wet and dry cutting condition. All 
Ahmed : Effect Of Wet And Dry Cutting Conditions On Dimensional Accuracy In Turning

experiments were done at depth of cut of $(0.6 \mathrm{~mm})$ on Takisawa turning machine as shown in Fig. (2). A profile projector device (VRZ-U) with magnification (10X) was used as an untouched measuring device with precision $(0.0005 \mathrm{~mm})$, to measure the diameter of the work-piece in several points as shown in Fig.(3). The deviation in diameter of the job was precisely measured along its axis after one full pass of the machining over $(300 \mathrm{~mm})$ length. Water-Soluble oil lubricant was used as a cutting fluid in the wet cutting condition experiments, with flow rate about (1.717 litter/min). The oil was a commercial type with mixture ratio about (1:20 oil-water) according to the recommendation of it. Dimensional deviation is calculated by the deference between the diameter measured from initial and end of the machined work piece. The magnitude of cutting velocities, depth of cut and feed rates were taken in the standared permisible cutting range for finishing operation of medium carbon steel by high speed steel cuting tool [8]. The experimental process were done at the following cutting conditions.

*Cutting Velocity V: $(33,47,70 \& 90 \mathrm{~m} / \mathrm{min})$.

*Depth of cut: $(0.6 \mathrm{~mm})$.

*Feed Rate f: $(0.097,0.19,0.28 \& 0.39 \mathrm{~mm} / \mathrm{rev})$.

*The experimentes were done at wet cutting condition and repeated in dry cutting condition.

*Work piece was held between two centers in order to become the initial and end of the work piece in the same force applied and bending conditions. Without suportes becouse its length only $(35 \mathrm{~cm})$ and over hang length of the tool is $(30 \mathrm{~mm})$ in all experiments.

*Tool Angles: As in the following table (2) and Fig. (1)

Table (1), Chemical composition of work piece material.

\begin{tabular}{|c|c|}
\hline Element & Composition \\
\hline Carbon $(\mathrm{C})$ & $0.35 \%$ \\
\hline Impurities* & $0.05 \%$ (Can not inspected) \\
\hline Iron $(\mathrm{Fe})$ & Balance \\
\hline
\end{tabular}

Table (2): Tool point angles for HSS cutting tool [8].

\begin{tabular}{|ll|c||}
\hline \multicolumn{2}{|l|}{ Angles description } & Angles \\
\hline Back rake angle & $\boldsymbol{\gamma}_{\mathbf{B}}$ & $10^{\mathbf{}}$ \\
\hline Side rake angle & $\boldsymbol{\gamma}_{\mathbf{S}}$ & $12^{\mathbf{}}$. \\
\hline Side cutting edge & $\boldsymbol{\theta}_{\mathbf{s}}$ & $15^{\mathbf{o}}$ \\
\hline End cutting edge & $\boldsymbol{\theta}_{\mathbf{e}}$ & $15^{\mathbf{o}}$ \\
\hline Side relief angle & $\boldsymbol{\alpha}_{\mathbf{s}}$ & $5^{\mathbf{o}}$ \\
\hline End relief angle & $\boldsymbol{\alpha}_{\mathbf{e}}$ & $5^{\mathbf{0}}$ \\
\hline
\end{tabular}

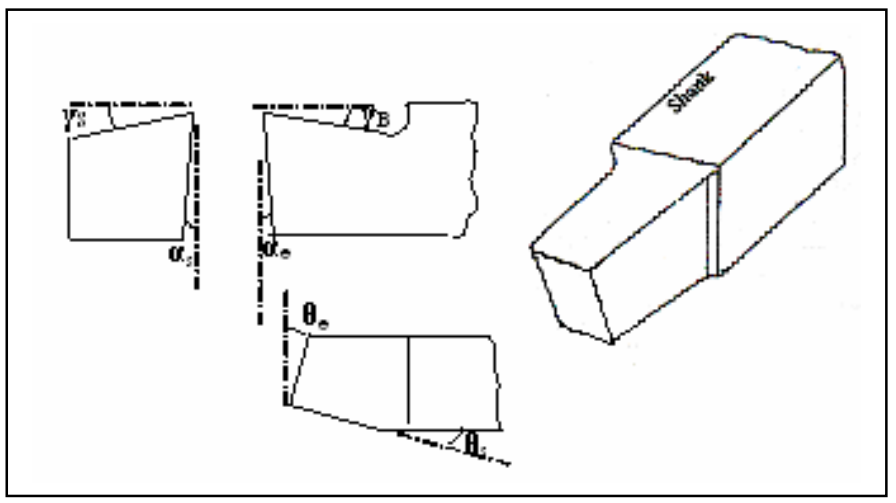

Fig. (1) Designations and symbols for a right hand high speed steel cutting tool [8]. 


\begin{tabular}{llll} 
Al-Rafidain Engineering & Vol.15 & No.4 & 2007 \\
\hline
\end{tabular}

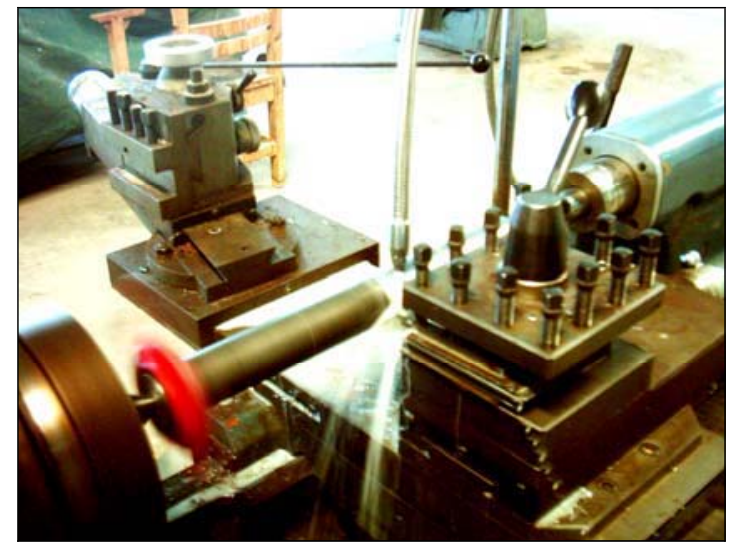

Fig.(2) Photographic view of the experimental set up for wet turning steel on TAKISAWA lathe machine.

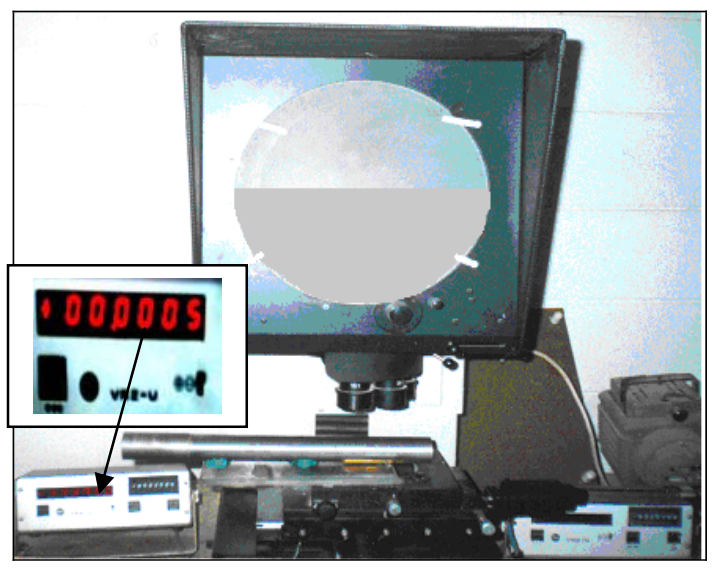

Fig.(3) Profilemeter with high precision digitally measurement. (Appearing smallest length can be measured).

After the experiments were done the dimensional deviation is calculated by the difference between the diameters measured at the initial and end of the same work piece and the initial diameter was taken as a desired diameter and the diameters measured in other places on the same work piece were compared with it. The results were recorded in the table (3).

Table (3): Dimensional deviation (DD) of specimens in different cutting parameters.

\begin{tabular}{|c|c|c|c|c|c|c|c|}
\hline $\begin{array}{c}\mathrm{V} \\
(\mathrm{m} / \mathrm{min})\end{array}$ & $\begin{array}{c}\mathrm{f} \\
(\mathrm{mm} / \mathrm{rev})\end{array}$ & $\begin{array}{c}\text { Wet } \\
\text { cutting } \\
\mathrm{DD}(\mathrm{mm})\end{array}$ & $\begin{array}{c}\text { Dry } \\
\text { cutting } \\
\mathrm{DD}(\mathrm{mm}) \\
\end{array}$ & $\begin{array}{c}\mathrm{V} \\
(\mathrm{m} / \mathrm{min})\end{array}$ & $\begin{array}{c}\mathrm{f} \\
(\mathrm{mm} / \mathrm{rev})\end{array}$ & $\begin{array}{c}\text { Wet } \\
\text { cutting } \\
\mathrm{DD}(\mathrm{mm})\end{array}$ & $\begin{array}{c}\text { Dry } \\
\text { cutting } \\
\mathrm{DD}(\mathrm{mm})\end{array}$ \\
\hline \multirow{4}{*}{33} & 0.097 & 0.027 & 0.02 & \multirow{4}{*}{70} & 0.097 & 0.048 & 0.025 \\
\hline & 0.19 & 0.02 & 0.016 & & 0.19 & 0.0405 & 0.0235 \\
\hline & 0.28 & 0.015 & 0.009 & & 0.28 & 0.031 & 0.015 \\
\hline & 0.39 & 0.0115 & 0.0095 & & 0.39 & 0.0245 & -0.0005 \\
\hline \multirow{4}{*}{47} & 0.097 & 0.0445 & 0.0305 & \multirow{4}{*}{90} & 0.097 & 0.0615 & -0.0025 \\
\hline & 0.19 & 0.0395 & 0.0265 & & 0.19 & 0.043 & -0.005 \\
\hline & 0.28 & 0.0235 & 0.015 & & 0.28 & 0.042 & -0.0085 \\
\hline & 0.39 & 0.0185 & 0.0055 & & 0.39 & 0.036 & -0.01 \\
\hline
\end{tabular}

\section{Results and Discussion}

Cutting tool may fail by brittle fracture, plastic deformation or gradual wear [3]. In this work a new sharpened tool for each experiment was used and in all experiments and for both cases a gradual wear was observed. With progress of machining the tools attain crater wear at the rake surface and flank wear at the clearance surface. In order to inspect the dimensional deviation along the length of the work piece four 
Ahmed : Effect Of Wet And Dry Cutting Conditions On Dimensional Accuracy In Turning

machining work pieces were selected and diameters at five equispaced length were measured. The dimensional deviation along each work piece length was obtained as shown in Figs.(4 \& 5).The growth of average flank wear with machining length is translated as a dimensional deviation on the job. It is observed that with the progress of the cutting length the dimensional deviation increases along the length of the work piece. In wet cutting condition a larger dimensional deviation is observed due to the flank wear of the cutting tool which decreases tool length and increases of job diameter and, wet cooling prevents thermal expansion of the cutting tool. But, in dry cutting condition due to the thermal expansion of the cutting tool which substitutes this decrease in length of the cutting tool due to the flank wear and causes more accuracy obtained in diameter. In spite of high temperature and more tool wear is taken place in dry cutting condition but each phenomenon act in two opposite direction on the dimension of the work piece. The heat generation causes thermal expansion of the cutting tool which causes more metal removal and flank wear of the cutting tool edge due to the friction and high temperature causes decrease of the cutting tool and less metal removal. In Fig.(5) a negative deviation in dry cutting is observed due to the more thermal expansion in cutting tool in cause of higher velocity. Because the increase of the cutting velocity, it causes higher temperature and heat generation [4]. In Nikhils work [3] is observed that the dimensional deviation and surface roughness in dry and wet cutting condition was near to each other and in dry cutting condition the dimensional deviation were smaller than the wet cutting condition. Therefore he concluded that the traditional cooling fluid is not significant in metal cutting. In this work also observed that the dimensional deviation in dry cutting condition is less than the dimensional deviation in wet cutting condition. Fig.(6) Explains the effect of feed rate on the dimensional deviation as well as the comparison between the dry and wet cutting condition. It is clear that with the increase of the feed rate decreases the dimensional deviation in wet and dry cutting condition because with the increase of the feed rate the machining time decreases for the same machining length in spite of the increase of cutting force due to the increase of the feed rate. Machining time plays a great role on the tool wear, flank wear increases with the increase of machining time as explained obviously in Nikhils work [3]. Also it is shown that the dimensional deviation on the dry cutting is less than in the wet cutting condition, therefore more accuracy is obtained in dry cutting condition because in wet cutting only the deviation due to the flank wear is appeared. But in dry cutting there are two different types of deviation flank wear error and thermal expansion error and these two errors are in opposite effect, as explained before. Flank wear error causes an increase in diameter and thermal expansion error causes a decrease in diameter and each of them substitutes each other. Therefore the dimensional deviation of the work piece in dry cutting condition is less than it in wet cutting condition. It is clear that the effect of cutting velocity differs from dry and wet cutting condition. The increase of cutting velocity increases the dimensional deviation in wet cutting condition machining. But the increase of cutting velocity in dry cutting condition machining decreases the dimensional deviation due to the increase of the thermal generation and more thermal expansion of the cutting tool which substitutes the decrease of the cutting tool due to the flank wear occurred on the nose of the 


\begin{tabular}{llll} 
Al-Rafidain Engineering & Vol.15 & No.4 & 2007 \\
\hline
\end{tabular}

cutting tool. In Fig(6-D) the negative deviation at the end of the work piece is observed due to the high temperature and more thermal expansion of the cutting tool is occurred at cutting velocity $(90 \mathrm{~m} / \mathrm{min})$. In this case the expansion of the cutting tool is larger than the decrease of tool length due to the flank wear. Therefore a larger metal removal and the produced diameter is smaller than the desired diameter. With the increase of the cutting velocity, increases the gap between the curves, dry and wet cutting condition curve. It is clear that with the increase of the cutting velocity from $(33 \mathrm{~m} / \mathrm{min})$ in Fig.(6-A) to the $(90 \mathrm{~m} / \mathrm{min})$ in Fig.(6-D), the gap between the wet cutting condition and dry cutting condition curves increases and the dry cutting condition curves reach to the desired diameter or less. Thermal expansion of the cutting tool causes more metal removal and decreases the diameter of the job in dry cutting condition and at velocity of $(90 \mathrm{~m} / \mathrm{min})$ a large amount of heat is generated witch causes a large amount of thermal error larger than the error due to the flank wear. Therefore a work piece with diameter smaller than the desired diameter is obtained

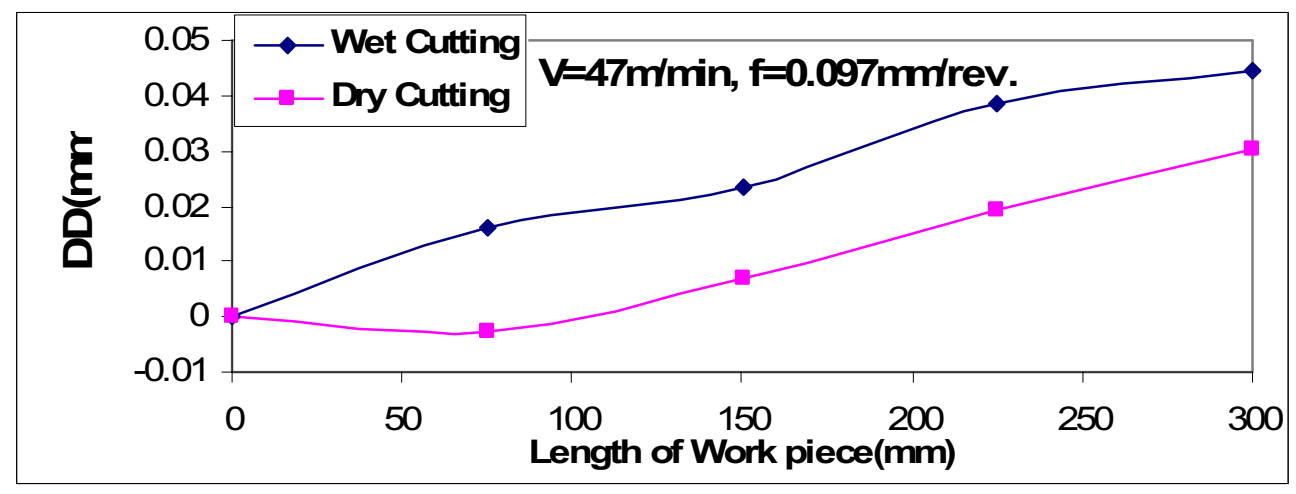

Fig.(4), Dimensional deviation (DD) along the length of the work piece in dry and wet cutting condition at velocity of $47 \mathrm{~m} / \mathrm{min}$ and feed rate of $0.097 \mathrm{~mm} / \mathrm{min}$.

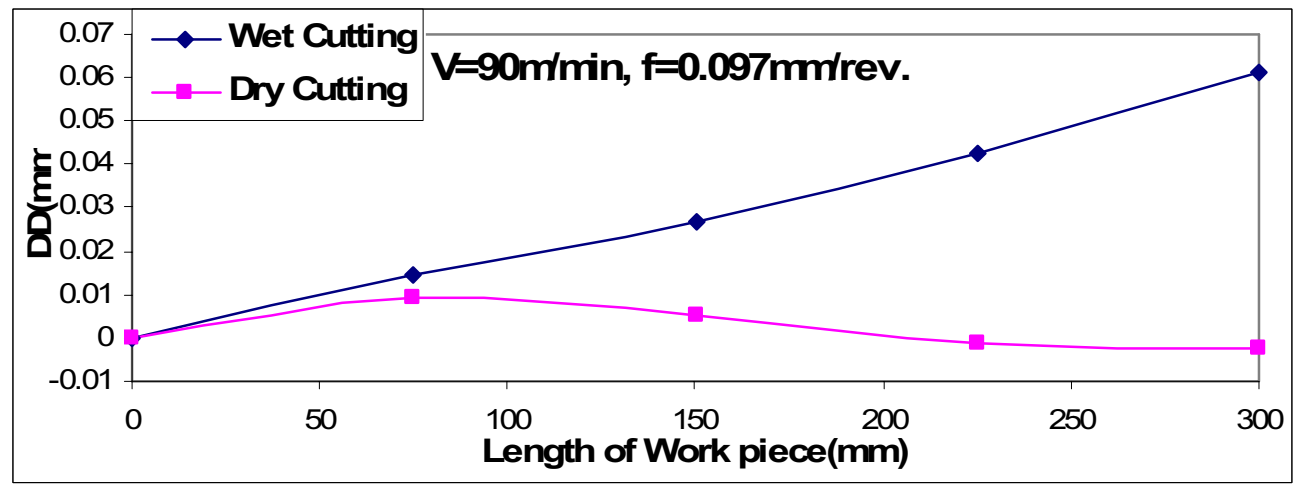

Fig (5) Dimensional deviation (DD) along the length of the work piece in dry and wet cutting condition at velocity of $90 \mathrm{~m} / \mathrm{min}$ and feed rate of $0.097 \mathrm{~mm} / \mathrm{min}$. 
Ahmed : Effect Of Wet And Dry Cutting Conditions On Dimensional Accuracy In Turning
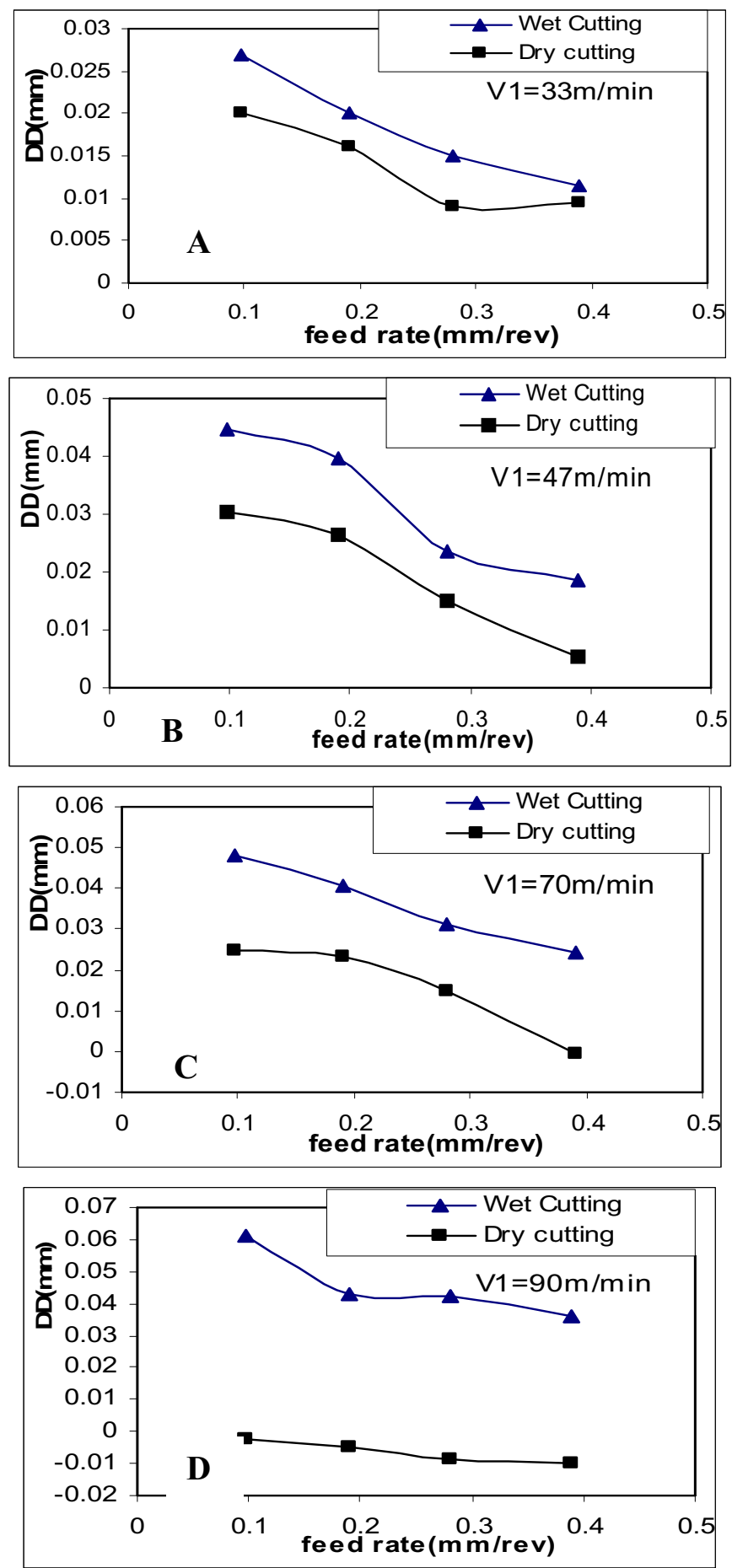

Fig.(6) Effect of feed rate on the Dimensional deviation (DD) at depth of cut $0.6 \mathrm{~mm}$ and velocities A) $33 \mathrm{~m} / \mathrm{min}$, B) $47 \mathrm{~m} / \mathrm{min}$, C) $70 \mathrm{~m} / \mathrm{min} \&$ D) $90 \mathrm{~m} / \mathrm{min}$. 


\section{$\begin{array}{llll}\text { Al-Rafidain Engineering } & \text { Vol.15 } & \text { No.4 } & 2007\end{array}$}

\section{Conclusions:}

In this work the following points are concluded:

1) Diameter of the work pieces machined in dry condition is more accurate than machined in wet cutting condition.

2) The increase of the feed rate decreases the dimensional deviation in both dry and wet cutting condition.

3) The effect of the increase of the cutting velocity has a different effect on the dimensional deviation in wet and dry cutting condition.

4) The increase of the cutting velocity increases the dimensional deviation in wet cutting condition, but decreases the dimensional deviation of the work pieces produced by the dry cutting condition.

5) The increase of the cutting velocity in dry cutting condition in permissible ranges provides the dimensional accuracy and do not cause any problem in diameter precision.

\section{References}

1) Marian Wiercigroch \& Erhan Budak, (Sources of nonlinearities chatter generation and suppression in metal cutting), The Royal Society, Phil. Trans. R. Soc. Lond. A (2001) 359, 663-693.

2) Tae, Jo Ko, Hee Sool Kim, and Bo Gu Chung, (Air-Oil Cooling Method for Turning of Hardened Material), The International Journal of Advanced Manufacturing Technology, Vol.15, No.7, July 1999.

3) Nikhil R. Dhar, Smaiya Islam, Md. Kamruzzaman \& Soumitra Paul, (Wear Behavior of Uncoated Carbide Inserts under Dry Wet and Cryogenic Cooling Conditions in Turning C-60 Steel), Journal of the Braz. Soc. Of Mechanical Sci. \& Eng., April-June 2006, Vol.XXVIII, No.2, pp. 146-152.

4) R. Azouzi and M. Guillot, (On-Line Prediction of Surface Finish and Dimensional Deviation in Turning Using Neural Network Based Sensor Fusion), Int. J. Mach. Tools Manufacture. Vol.37, No.9, 1997.

5) S.K. Choudhury, V.K. Jain, and S.Rama Krishna, (On-Line Monitoring of Tool Wear and Control of Dimensional Inaccuracy in Turning), Journal of Manufacturing Science and Engineering, Vol. 123, February 2001.

6) Twardowski Pawel, (Experimental Investigation Concerning Influence of Thermal Expansion Insert on Precision Turning of Hardened Steels), Tabor Czech Republic, June 2001.

7) Xiaoli Li and R. Du, (Analysis and Compensation of Work piece Errors in Turning), International Journal of Production Research, Vol.40, No.7, 2002.

8) Erope Kalpakjian, Steven R. Schmid, (Manufacturing Engineering and Technology), fourth edition, copyright 2001 by peavson education, Inc. 